\title{
Sistemas domóticos para adultos mayores con movilidad reducida
}

\author{
Ricardo Alarcón Contreras / Mónica Flores Marín
}

\section{Resumen}

Este artículo muestra la evolución de los sistemas de automatización para el hogar; la aparición de los estándares Zigbee, KNX y el protocolo X10. Recopila varios proyectos domóticos orientados a la asistencia del adulto mayor y explica la contribución de estos a su independencia. También se realiza una comparación de los estándares bajo diferentes parámetros y se concluye que para implementar estos proyectos es mejor la utilización de sensores y actuadores inalámbricos por no ser intrusivos. Siendo Zigbee la mejor opción por su costo, ahorro de energía, alcance, adaptabilidad, interoperabilidad y escalabilidad. En base a los casos de éxito mostrados y necesidades de los usuarios se proponen requerimientos mínimos para hogares inteligentes que brinden mayor independencia al adulto mayor.

\section{Palabras clave:}

Zigbee, X10, KNX, domótica, adulto mayor, sensores, independencia.

\section{Abstract}

This article shows the evolution of automation systems for home; the emergence of Zigbee, KNX and X10 protocol standards. Collects various automation projects aimed at assisting the elderly and explains the contribution of these to their independence. A comparison of the standards under different parameters is also performed and concludes that to implement these projects systems with wireless sensors and actuators are used for not being intrusive. Zigbee being the best choice for its low cost, energy saving, scope, adaptability, interoperability and scalability. Based on the success stories shown and user needs minimum requirements for smart homes that provide greater independence for the elderly are proposed.

\section{Keywords:}

Zigbee, X10, KNX, smart home, elderly, sensors, independence. 


\section{Introducción}

El envejecimiento afecta a la persona, familia y comunidad, por ello es multidimensional. La expectativa de vida en el Ecuador ha aumentado de 48.3 años en la década de 1950 a 76.5 años en la actualidad, esto se debe a la mejora en los sistemas de salud y trato de enfermedades. Este factor, junto con la disminución de natalidad, está produciendo un aumento en la población de la tercera edad a nivel mundial, en el país se espera que este grupo de personas alcance el $18 \%$ de la población total para el 2050. Por ello es necesario proponer respuestas integrales que ayuden a los mayores a continuar su vida de forma activa, tranquila y saludable (Ministerio de Inclusión Económica y Social [MIES], 2012).

Con el aumento de la edad surgen enfermedades y los familiares no permiten al adulto mayor vivir solo (Gaddam, Mukhopadhyay y Sen Gupta, 2009). Sin embargo al tratar de mantener su independencia la persona corre peligro al lidiar con actividades diarias debido a problemas en: sus sentidos, inmovilidad, aislamiento, necesidad de medicación constante y la posibilidad de caídas. Se sabe que tres cuartas partes de los adultos mayores tienen problemas de movilidad, el $50 \%$ auditivos, y aunque el $80 \%$ tiene una visión razonablemente buena, el $36 \%$ tiene un desorden fisiológico que puede degenerar su visión central (Demiris et al. , 2004).

Esta realidad ha incrementado el interés por sistemas de supervisión continua del bienestar de los adultos mayores, para reconocer su estado de salud tempranamente, notificar a los cuidadores en caso de emergencia $e$ incluso encontrar relaciones entre estilo de vida y salud (Raad y Yang, 2008) haciendo posible atenderlos de mejor manera. (Suryadevara, Mukhopadhyay, Rayudu y Huang, 2012). Este tipo de vigilancia se puede lograr mediante la implementación de sistemas inteligentes, que recopilen información de forma no intrusiva. (Viani et al., 2013).

Por lo tanto esta investigación tiene como objetivo mostrar algunos estándares y protocolos de automatización existente para compararlos y encontrar el más viable para nuestro medio según las necesidades de los adultos mayores, y por medio de casos de éxito proponer los requerimientos teóricos mínimos de una casa inteligente para su supervisión y asistencia.

\section{Marco Teórico}

En el desarrollo de este documento se presenta los conceptos relacionados con el adulto mayor, su independencia e inconvenientes de la edad, junto con un recuento del desarrollo de la domótica y el funcionamiento de algunos de sus estándares.

\section{Situación de los adultos mayores}

El envejecimiento es un proceso natural que afecta a todas las personas durante el cual se evidencia una disminución en las capacidades físicas, mentales y sociológicas (Dueñas, 2012) cuyo impacto varía según su entorno social y geográfico. Es necesario llevar a cabo una valoración para desarrollar un plan para el cuidado de la persona que vaya acorde a su grado de independencia (Ministerio de Salud Pública [MSP], 2011), que de acuerdo al MSP es "la habilidad de un individuo para actuar y funcionar en la vida diaria, con autonomía e independencia" (MSP, 2008, p. 31).

La independencia de la persona está asociada a su nivel de funcionalidad que se define dentro de tres componentes relacionadas al individuo: las actividades básicas de la vida diaria (ABVD) como alimentarse, asearse y movilizarse dentro del hogar. Por otro lado están las consideradas como instrumentales de la vida diaria (AIVD), que implican un desenvolvimiento en tareas un poco más complejas, como movilizarse fuera de la casa y realizar labores domésticas sencillas, finalmente tenemos la movilidad avanzada de la vida diaria (AAVD) que evalúan la fuerza 
para levantar objetos o agacharse (MSP, 2011; Arroyo et al. , 2007).

En base a la dificultad que presenta el adulto mayor para llevar a cabo ABVD, AIVD o AAVD es posible realizar una valoración que permita conocer el grado de dependencia funcional y su nivel de alteración según las actividades que el individuo es capaz de realizar (Arroyo et al. ,2007). La alteración leve implica dificultad para moverse largas distancias o subir escaleras; la alteración moderada, supone dificultad para movilizarse dentro del hogar, y la alteración severa, implica dificultad para movilizarse independientemente de la cama para realizar las $A B V D$, hasta la inmovilidad total (Barrantes-Monge, García-Mayo, Gutiérrez-Robledo y Miguel-Jaimes, 2007).

Las limitaciones funcionales merman la calidad de vida de los adultos mayores; por ello el objetivo es buscar maneras de ayudarlos a tener un envejecimiento saludable (MIES, 2012). Por lo tanto se propone el uso de sistemas domóticos para ayudar a los ancianos que padecen alteraciones leves o moderadas a recuperar su independencia dentro del hogar en situaciones de movilidad reducida (Demiris et al., 2004).

A nivel poblacional, en el Ecuador, las personas mayores a 65 años representan el $7 \%$, de los cuales $49 \%$ vive acompañado de su hijo o hija, $16 \%$ con el nieto y $15 \%$ esposo o compañero (INEC 1,2010 ). Por consiguiente aproximadamente el $65 \%$ vive con alguien que se hace cargo de ellos.

Para estas personas sería útil tener la posibilidad de vigilar a sus familiares (Raad y Yang, 2008) además de saber que en casa tienen como realizar sus actividades con mayor facilidad, seguridad e independencia cuando ellos no están, lo que es posible mediante sistemas domóticos (Demiris et al., 2004; Gaddam et al. , 2009).

\section{Necesidades de los adultos mayores \\ Entre los principales aspectos de interés de}

I Instituto Nacional de listndisticas y (Censess las personas de la tercera edad, de acuerdo a un grupo focal realizado por Demiris et al. (2004), se encuentran: la asistencia visual y auditiva, ayuda de emergencia, prevención y detección de caídas, iluminación automática, revisión de parámetros fisiológicos como niveles de glucosa y presión sanguínea, control de seguridad del horno y la estufa, seguridad del hogar, detección de intrusos y recordatorios de eventos por venir en su agenda. Por otro lado al consultar a los participantes del estudio sobre los sistemas de automatización para su asistencia, existe la preocupación de la privacidad, la posibilidad de que los cuidadores sean reemplazados, la facilidad para usar y la necesidad de capacitación para el uso de estos sistemas. Este estudio coincide con Collins, Bhatti, Dexter y Rabbitt (1993) en que no encontraron relación alguna entre la edad y la fobia a la tecnología, pero aclaran que el estudio se hizo con personas de clase media con buen nivel de educación.

El inconveniente que existe al momento de desarrollar sistemas domóticos es que su objetivo es lograr avances en investigación a nivel tecnológico y no se centran en el adulto mayor, como resultado las interfaces no pueden ser empleadas completamente (Demiris et al., 2004).

\section{Evolución de la domótica}

En la década de los 80 se desarrolló gran cantidad de proyectos en distintas partes del mundo, en busca de un estándar para el control automático de viviendas inteligentes; el objetivo era que los dispositivos interconectados reúnan los requerimientos mínimos para lograr un estilo de vida más cómodo, seguro, económico, conveniente y satisfactorio (Murata, Namekawa y Hamabe, 1983).

En Japón, en 1981, se juntaron siete empresas con dos universidades para crear un estándar para Home Bus System (HBS) que permitiera emplear dispositivos de distintos fabricantes que trabajen bajo un mismo protocolo o esquema de comunicación. El Hardware de HBS se resume en 4 puntos: un sistema de cableado simple, salidas de 
televisión mejoradas usadas como salidas de información, aprovechar el cableado interno del hogar sin mayores modificaciones y la construcción de interfaces LSI a bajo costo (Hamabe, Murata y Namekawa, 1986).

En la misma época en Estados Unidos la Asociación Nacional de Constructores (NAHB ${ }^{2}$, por su nombre en inglés), en 1984, compuesta por grandes compañías como Emerson, AT\&T, Telecom, General Electric entre otras, iniciaron el proyecto Smart House que apuntaba a desarrollar la casa inteligente. Para lograrlo construyeron dos casas de laboratorio en 1987, en 1989 esperaban tener 100 unidades demostrativas, y el objetivo final era tener viviendas inteligentes comerciales para inicios de los 90 (Edgar, 1987). Junto con el proyecto se lanza Consumer Electronic Bus (CEBus) para la comunicación entre los módulos de la Smart House, basado en el modelo de interconexión ISO/OSI ${ }^{3}$ y como medio de transporte de datos podía ser par trenzado, infrarrojo, radio frecuencia, cable coaxial o fibra óptica (Douligeris, Khawand y Khawand, 1991).
Skrzypczak (1987) hizo una prospección de lo que sería una casa inteligente en el 2010, a nivel de funcionamiento pensaba en el uso de un procesador central que manejase los demás dispositivos del hogar; pero condicionó este avance al desarrollo de la informática con su capacidad para procesar la información y la ergonomía, refiriéndose al desarrollo de interfaces acorde a las necesidades humanas (Skrzypczak, 1987, p. 82).

En el continente Europeo también se desarrollaron proyectos de automatización de viviendas como el Sistema de hogar interactivo (IHS, por su nombre en inglés) liderado por empresas como Siemens, Electrolux y Philips, entre otras; buscando la creación de un estándar para conectar las aplicaciones domésticas a un bus de datos común y que permita utilizar distintos medios de interconexión. A pesar de que los servicios manejados en el hogar se podían transmitir por cualquiera de los medios, los límites venían dados por el ancho de banda requerido; la tabla 1 muestra un resumen de las clases junto con los medios de transmisión que la soportan (Tritton, 1988).

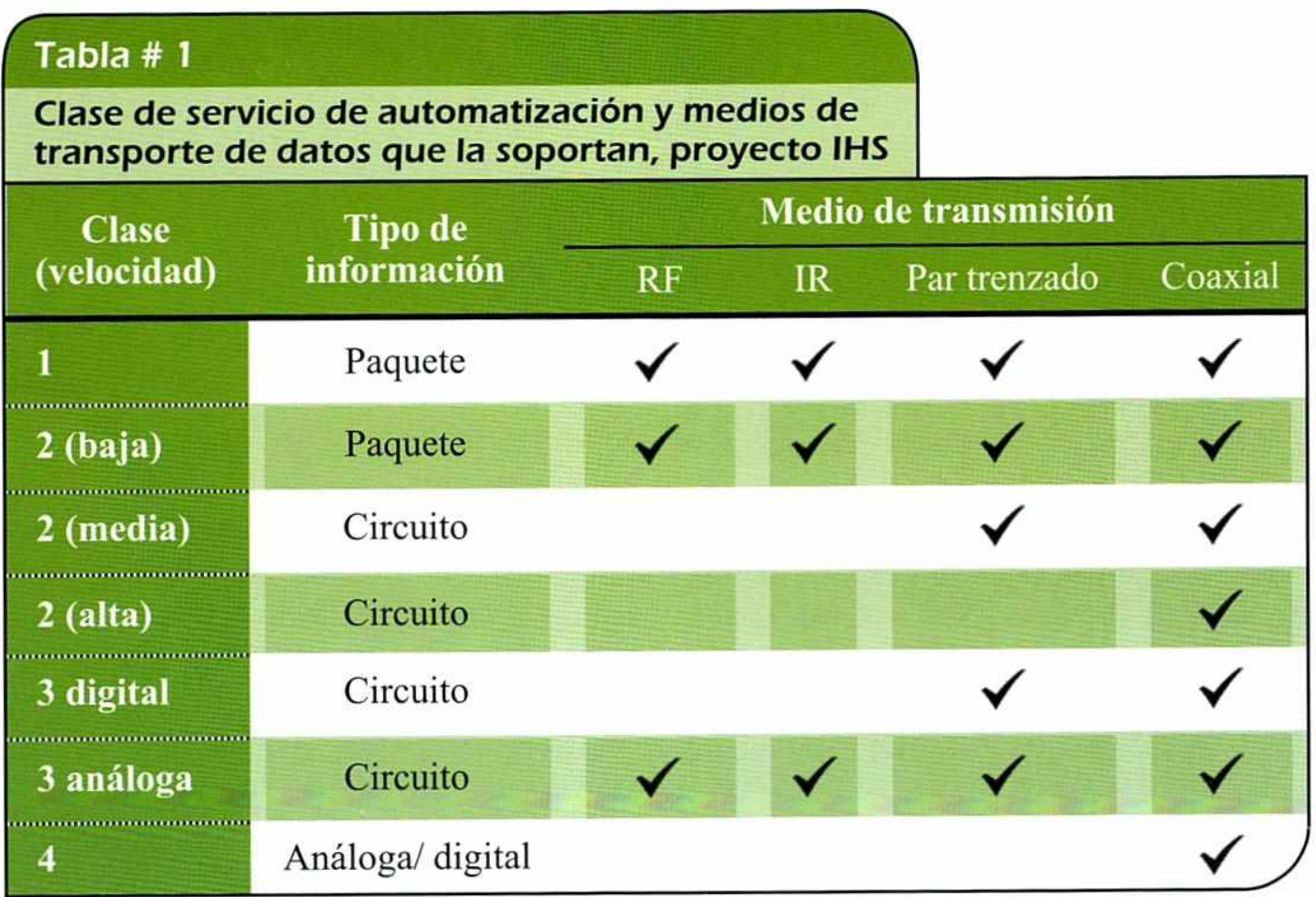

Nota: RF: Radio Frecuencia, IR: Infrarrojo. Adaptado de Tritton, J. A. (Junio, 1988). Interactive Home Systems (IHS)-an overview. Private Switching Systems and Networks, 1988., International Conference, 195-200.

${ }^{2}$ National Association of Home Builders

3 Open System Interconection 
De acuerdo a Domínguez y Sáez (2006) para comprender mejor la domótica es necesario entenderla como la combinación del uso de dispositivos electrónicos y la interacción de sistemas complejos ${ }^{4}$ en el hogar, que se traduce en el componente social y los sistemas tecnológicos utilizados para su automatización. En un principio la domótica se pensó como respuesta a las necesidades de gestionar distintas actividades que se realizan en las viviendas, como el manejo de energía, seguridad y confort; sin embargo hoy en día se la entiende como automatización y control abriendo el abanico de posibles aplicaciones (Junestrand, Pasarte y Vázquez, 2005).

\section{Estándares y protocolos de automatización}

Para lograr la implementación de arquitecturas e infraestructuras de un ambiente inteligente; es recomendable que sean escalables, de fácil adaptabilidad, seguras al momento de transmitir información en la red interna y que exista apertura al uso de dispositivos de distintos fabricantes; para lograrlo es necesario abstraer la comunicación de los elementos que se utiliza (Fabbricatore, Zucker, Ziganki y Karduck, 2011). Entre los principales estándares que se han ido desarrollando para el propósito de hogares inteligentes están el KNX y Zigbee además del protocolo X10.

\section{Protocolo X10}

$\mathrm{X} 10$ fue el primer protocolo con fines de automatización, creado en el año de 1975 basado en corrientes portadoras, su accesibilidad y sencillez de implementación le dio gran acogida en Estados Unidos por lo que hoy en día se continúa fabricando dispositivos basados en él (Gill, Shuang-Hua Yang, Fang Yao y Xin Lu, 2009). La desventaja que presenta es el uso de corrientes portadoras, pues depende mucho de la calidad con que estas llegan a las casas por lo que presen-

T'L'n sistema complejo es aquel constituido por un yran nuinero de partes que interactúan en una forma no scneilla... Dadas las propiedades de las partes $y$ las leyces de interacción, no es un prublema trivial inferir las propiedades del todo".(Siez Vacas Iः. , 199() ta problemas de interferencia (Flores y Rosero, 2014).

\section{Estándar $\mathrm{KNX}$}

$\mathrm{EIBA}^{5}, \mathrm{EHS}^{6}$ y $\mathrm{BCI}^{7}$, se unieron para desarrollar KNX que permiten la interconexión de dispositivos independientemente del fabricante. Entre sus principales características está el uso de un medio para la transmisión de información dedicado a la red de automatización, sea por par trenzado, radio frecuencia o línea de fuerza. Los actuadores y sensores se conectan a un BUS de datos y permite el control de una amplia gama de servicios. Este estándar ha logrado reunir distintas normas como ISO/IEC 14543-3 a nivel internacional, CENELEC EN 50090 y CEN EN 13321-1 como estándar europeo e incluso como estándar nacional en países como China bajo la norma GB/T 20965. Presentando como parte de sus ventajas la interoperabilidad de sus elementos y la escalabilidad, el único requisito es el uso de ETS $^{8}$ para el diseño, implementación y configuración de productos que trabajen bajo este estándar (KNX Association, 2014).

A nivel de funcionamiento es un sistema descentralizado, pues la "inteligencia" está distribuida en los dispositivos. La red diseñada para KNX es de tres niveles, en el primero están las líneas principales que dividen cada área del sistema según lo requiera el diseño del proyecto. De ellas se desprenden las líneas secundarias a las cuales se conectan los dispositivos del bus o subscriptores que pueden ser un máximo de 64 por línea conformando. $Y$ en el tercer nivel, para la interconexión entre niveles se utiliza acopladores de línea, además acepta otras topologías de red, como de estrella, mas no de anillo. La figura uno muestra un ejemplo de una red de sistema KNX. (ABB, 2014; KNX Association, 2014 ; Synco,2013)

\footnotetext{
Asenciación de instalación de Bus liuropea (I:ureppean installation Bus Association)

"Sistema de luygar curopeo (Iuropean Home System)

- Club Internacional BatiBL'S (13ntil3L 'S Club Iniemational)

* Iinginecring Tool Softuarc, herramienta para cl diserio, la implementacién

I la contiguracién de la instalacion que posea productos certificados $\mathrm{K} N \mathrm{NX}$ (แาพw:knx.ong)
} 


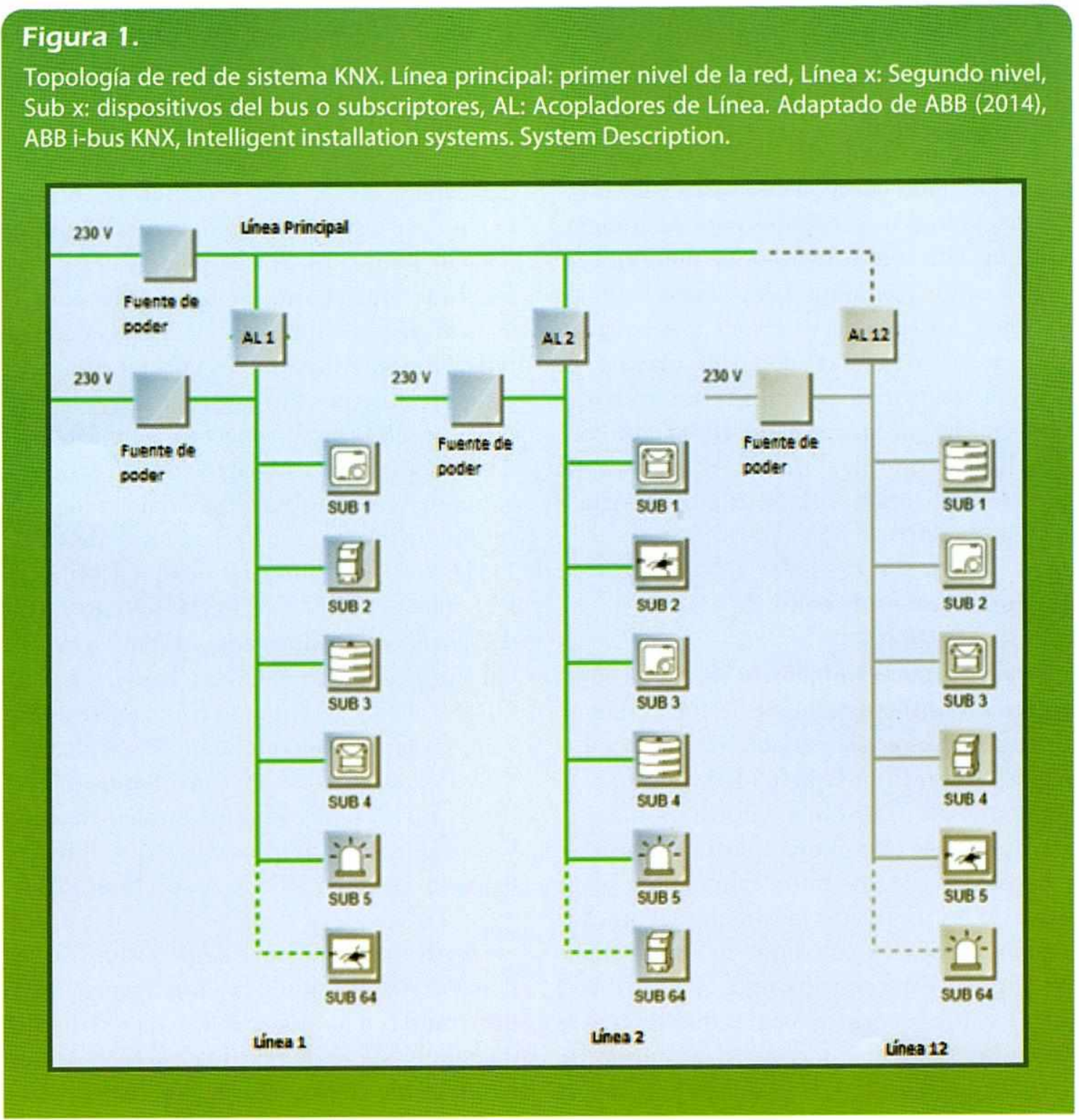

\section{Estándar Zigbee}

El estándar IEEE 802.15.4, conocido como Zigbee, está compuesto por un conjunto de protocolos para el desarrollo e implementación de redes de corto alcance y baja velocidad de transmisión de datos, con una tasa de hasta $250 \mathrm{kbps}$. Opera en las bandas $860 \mathrm{MHz}, 915 \mathrm{MHz}$ y $2.4 \mathrm{Ghz}$ y usa un medio inalámbrico, a través del cual los datos viajan encriptados por seguridad. Su objetivo es tener bajo consumo de energía para utilizar equipos a batería, bajo costo tanto de productos como su instalación y mantenimiento, además de ser de corto alcance. Este estándar es uno de los más usados en la actualidad; parte de sus fortalezas es la interoperabilidad entre los dispositivos de los fabricantes que pertenecen a la Alianza ZigBee. Además sus elementos son plug and play (Zigbee Alliance, 2014).

Es un protocolo de malla, todos los dispositivos se interconectan entre sí, por lo que cada elemento de la red puede servir como repetidora y receptor a la vez lo que permite aumentar la distancia de alcance en el envío de datos, pues la información "salta" entre los nodos hasta 6 veces antes de que esta se deseche. Al ser un estándar abierto permite construir prototipos a medida sin ser de un fabricante específico (Withanage et al. , 2014). 
Este estándar ofrece tres especificaciones distintas según el requerimiento del usuario, la convencional y más utilizada Zigbee PRO que permite crear una red de malla de bajo costo. También bajo consumo de energía o incluso utilizar dispositivos autoalimentados con Green Power ${ }^{9}$, que implementa redundancia y autorregulación y finalmente la Zigbee RF4CE fue desarrollada para implementaciones que requieren interacción sencilla de dispositivo a dispositivo y no las demás bondades de crear una red de automatización completa. La red se compone por tres tipos de instrumentos, los coordinadores que se encargan de la formación y seguridad de la misma, enrutadores que permiten aumentar su alcance y dispositivos finales que son los sensores y actuadores. Además es posible usar uno de los coordinadores como puerta de enlace al internet permitiendo acceso remoto, la figura 2 muestra un diagrama que ejemplifica la topología de este estándar (Zigbee Alliance, 2014).

\section{Figura 2.}

Ejemplo de topología de red Zigbee. Adaptado de Zigbee Alliance (2014).

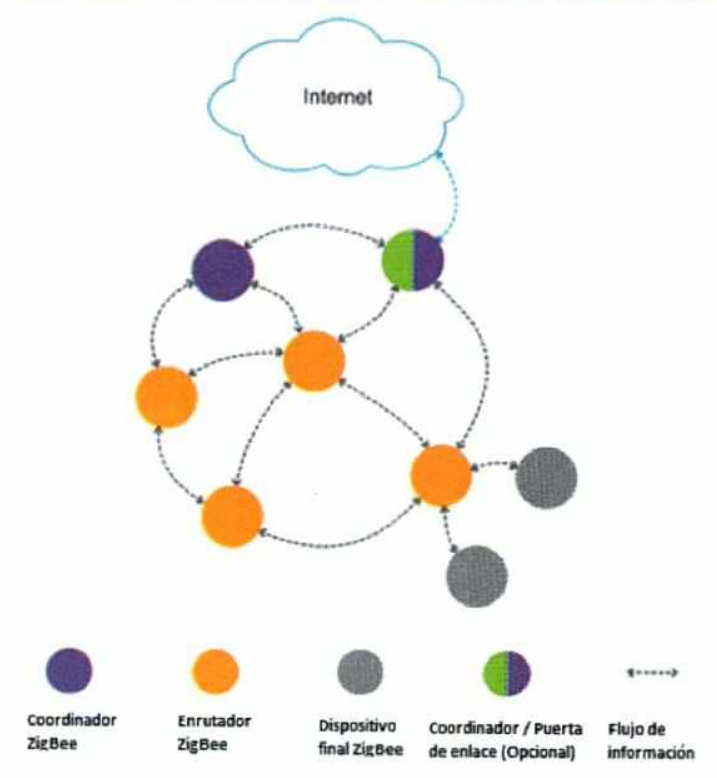

${ }^{9}$ Green Power es una caracteristica implementada en el 2012 que permite a los dispositivos trabajar sin bateria y obtienen la energia aprovechando la vibración, luz y movimiento. El consumo aproximado es de 200 micro joules contra los 20 mili Joules de la especificación convencional de Zigbee y 2 kilo Joules que necesita Wifi (Zigbee Alliance, 2014)
Más allá de que este estándar podría presentar fallas en algunos casos, su sencillez de implementación y autoorganización de la red le han dado gran popularidad para la creación de redes de sensores inalámbricos (WSN, por su nombre en inglés). Se componen de numerosos dispositivos de bajo consumo de energía, distribuidos espacialmente a lo largo de la vivienda, permitiendo saber dónde se encuentra la persona y las rutas que recorre dentro del hogar. La implementación de WSNs ha ganado popularidad debido a que no necesitan modificar la construcción y recopila información de forma no intrusiva (Viani et al. ,2013).

\section{Casos de Éxito}

A continuación se incluyen casos de éxito que implementaron sistemas domóticos para asistencia de adultos mayores utilizando los estándares mencionados anteriormente.

\section{Proyecto A}

Gaddam, Mukhopadhyay y Sen Gupta (2011) realizaron un proyecto domótico empleando una WSN, que enviaba un mensaje de texto en caso de anomalías. Utilizaba sensores que median el tiempo de uso de electrodomésticos, cama y agua, también incluyeron un botón de pánico para el envío de mensajes en caso de emergencia. La información de los sensores se transmitían a una computadora con un software que bajo parámetros predefinidos alertaba la existencia de comportamientos fuera de lo común, además de llevar un registro del uso de los sensores los cuales se interconectaban con Zigbee implementado por medio de una tarjeta. Durante las pruebas encontraron que la confiabilidad de la transmisión decaía al aumentar la distancia entre dispositivos, alcanzando un $99 \%$ de efectividad hasta los 18 metros y cayendo a $91.67 \%$ a los 26 metros. 


\section{Proyecto B}

Suryadevara et al. (2012). Sugieren un sistema de monitorización compuesto por tres niveles, donde el primero comprende los sensores como medidores de presión para saber si está en uso la cama, silla o sofá; en los tomacorrientes se adaptan dispositivos que permiten saber si está utilizando los electrodomésticos, además se distribuyeron varios botones de pánico a lo largo de la vivienda, todos estos elementos se comunican a través de módulos Zigbee. El segundo nivel recopila información de los sensores y los guarda como eventos en la computadora a la vez que muestra en la interfaz el estado de los mismos en tiempo real, el último nivel se encarga de la toma de decisiones en base a dicha información. Con estos datos se calculan indicadores de bienestar de la persona según el uso o inactividad de las dependencias del hogar; complementado con el cálculo de cuánto tiempo tarda en realizar sus actividades, el cual es comparado con un valor estimado que debería completarlas; de esta forma es posible conocer si la persona anda bien o necesita asistencia para sus ABVD o AIVD.

En un estudio posterior Suryadevara, Mukhopadhyay, Wang, Rayudu y Huang (2013) realizaron una modificación en la determinación de los índices de bienestar; cambiando el uso de parámetros definidos, por proyecciones del tiempo que normalmente le toma al adulto mayor completar sus actividades para compararlo con las lecturas diarias, de esta forma los resultados eran más precisos para cada individuo.

\section{Proyecto C}

Para los casos de adultos mayores con enfermedades crónicas es posible implementar un sistema de supervisión con tres tipos de nodos: sensores corporales para revisar signos vitales, sensores en el ambiente y nodos de acceso que permiten conectarse a la red telefónica por medio de internet; de esta forma se puede conocer el estado de salud de la persona y en caso de emergencia enviar la alerta. La comunicación entre sensores se da a través de Zigbee, pues no se requiere largo alcance (Popescu, Dobrescu, Maciuca y Marcu, 2012).

\section{Proyecto D}

Por su parte la Unión Europea ha conducido el proyecto Persona, que busca unificar distintas aproximaciones a la arquitectura de los sistemas, orientados a viviendas inteligentes que permitan desarrollar un ambiente de vida asistido para personas mayores, capaz de integrar los distintos servicios del hogar. El proyecto requiere una gran inversión para interconectar dispositivos, pero la posibilidad de trabajar sobre Zigbee la reduce considerablemente. Por motivos de interoperabilidad este proyecto posee una capa de integración que se abstrae de los sensores (SAIL ${ }^{10}$, por su nombre en inglés) que da la posibilidad de operar en combinación con otros estándares como el caso de KNX (Fabbricatore et al. , 2011).

\section{Proyecto E}

Por otro lado Gill et al. (2009) proponen implementar un sistema con una red de automatización para las luces y calefacción, a la que se conecta un control remoto montada sobre Zigbee. Para los servicios que requieren una mayor tasa de transferencia de datos, como multimedia y conexión remota, a través de internet se usa una red Wifi, la interacción entre ambas ocurre por medio de una puerta de acceso intermedia.

\section{Proyecto F}

AlShu'eili, Sen Gupta y Mukhopadhyay (2011) diseñaron e implementaron un sistema de control de luces y electrodomésticos por comandos de voz, que consta de tres módulos: un micrófono portátil que incluye un módulo RF, un controlador central y controladores de electrodomésticos. Para su

\footnotetext{
"'scnsor Abstraction Integration I Ayer
} 
funcionamiento los comandos de voz son recibidos por el módulo del micrófono que los filtra y los convierte a una señal digital, la cual se envía utilizando Zigbee al computador central, para que la procese y encienda o apague los electrodomésticos según la orden dada por el usuario.

\section{Proyecto G}

Este proyecto emplea una aplicación para que un móvil con Android interactúe con un sistema KNX, empleando un módulo de control de acceso y uno de automatización. El módulo de control de acceso se encarga de verificar que el dispositivo móvil tiene los permisos para trabajar con el sistema. Por otro lado en el módulo de automatización se encuentra la aplicación para el teléfono y un programa hecho en JAVA para editar la configuración de dicha aplicación. Para poder utilizar el editor se debe activar un parche mediante la herramienta ETS de KNX, luego de esto el usuario puede seleccionar las pantallas y módulos KNX que desea controlar desde la aplicación, el resultado es un archivo que se

\section{Proyecto $\mathrm{H}$}

El sistema Glam i-homeCare de la Universidad de Glamorgan emplea redes de inteligencia artificial, empleando una computadora central que se conecta a servicios del hogar, como el de televisión, luces y aires acondicionados; por medio de interfaces X10 y a los sensores a través de IP; adicionalmente utiliza Bluetooth para conectarse a un cinturón capaz de medir parámetros fisiológicos de la persona. Este sistema domótico captura información del estado de los sensores cada 5 minutos y la va acumulando en el banco de experiencias para la retroalimentación.

Tiene como fin permitir al sistema aprender sobre el comportamiento del individuo, a la vez que permite a personas autorizadas a revisar la información recopilada sobre el paciente para un mejor cuidado. Este proyecto se encuentra en etapa de pruebas; por lo que actualmente contempla un hogar de un solo ocupante, ya que no maneja identificación de la persona (Mowafey y Gardner, 2013).

\begin{abstract}
importa a la App que convierte al celular en un control remoto del sistema domótico, que también funciona estando fuera de la vivienda a través de internet (De Luca, Lillo, Mainetti, Mighali, Patrono y Sergi, 2013). Nazabal, Matías, Fernández-Valdivielso, Falcone, Branchi y Mukhopadhyay (2012) utilizaron la interfaz KNXNet/IP para implementar este tipo de conexión.
\end{abstract}

\begin{tabular}{|c|c|c|c|c|}
\hline \multicolumn{5}{|c|}{$\begin{array}{l}\text { Deducción del estado de ánimo de una persona, en } \\
\text { base a la combinación de parámetros fisiológicos }\end{array}$} \\
\hline $\begin{array}{l}\text { Reglas del } \\
\text { sistema }\end{array}$ & $\begin{array}{l}\text { Ritmo } \\
\text { cardiaco } \\
\end{array}$ & $\begin{array}{l}\text { Ritmo } \\
\text { respiratorio } \\
\end{array}$ & Temperatura & Emoción \\
\hline 1 & Muy bajo & Muy bajo & Normal & Feliz \\
\hline 2 & Muy bajo & Bajo & Normal & Feliz \\
\hline 3 & Bajo & Muy bajo & Normal & Feliz \\
\hline 4 & Bajo & Bajo & Normal & Feliz \\
\hline 5 & Alto & Alto & Alto & Enojado \\
\hline 6 & Alto & Alto & Muy alto & Enojado \\
\hline 7 & Alto & Muy alto & Alto & Enojado \\
\hline 8 & Alto & Muy alto & Muy alto & Enojado \\
\hline 9 & Muy alto & Alto & Alto & Enojado \\
\hline 10 & Muy alto & Alto & Muy alto & Enojado \\
\hline 11 & Muy alto & Muy alto & Alto & Enojado \\
\hline 12 & Muy alto & Muy alto & Muy alto & Enojado \\
\hline 13 & \multicolumn{3}{|c|}{ Ninguna de las anteriores } & Neutral \\
\hline
\end{tabular}




\section{Metodología}

El procedimiento escogido para cumplir con el objetivo de esta investigación descriptiva no experimental de enfoque cualitativo que emplea como herramienta de recolección de datos la revisión documental, fue: realizar una investigación preliminar para conocer los estándares existentes de los cuales se seleccionaron tres, X10, KNX y Zigbee.

Luego de esto se tomó como guía los estudios hechos por Fabbricatore et al. (2011) y por Withanage et al. (2014) quienes indican que la forma de evaluar o seleccionar la tecnología para implementar sistemas domóticos es escasa. Proponen ciertos requerimientos para que el usuario tenga una mejor perspectiva. Los factores seleccionados fueron: costos de implementación y de equipos, lo que incluye el medio de transmisión, interoperabilidad, rango de alcance para la transmisión de datos, escalabilidad, seguridad en la transmisión de los datos, fiabilidad, facilidad de implementación y capacidad de controlar las bondades del hogar. Los resultados de dicha comparación permitirán definir el más idóneo para aplicaciones domóticas.

Finalmente en base a los casos de éxito de sistemas inteligentes que emplean los están- dares mencionados; se busca hallar tendencias que permitan definir los requerimientos mínimos para desarrollar un hogar domótico para la asistencia de adultos mayores, complementado con las necesidades de este sector de la población que mostró Demiris et al (2004) en su estudio. La principal herramienta de análisis a utilizarse son: las tablas que permiten resumir características y datos más relevantes para la investigación llevada a cabo; a la vez que se simplifica el establecimiento de las comparaciones propuestas para los distintos estándares y las similitudes entre los casos de éxito.

\section{Análisis de Resultados}

En la tabla 3 se muestra un resumen de los factores de comparación definidos en la metodología para cada uno de los estándares y el protocolo en cuestión. Además se incluyó el medio de transmisión, el cual influye de forma directa en los costos, puesto que el gasto es mayor cuando existe la necesidad de implementar cableado para el sistema; es por eso y debido al valor de sus dispositivos que KNX fue asociado a costos elevados (ABB, 2014). Por otro lado Zigbee no necesita implementar el medio de transmisión y X10 aprovecha el cableado eléctrico existente presentando costos menores.

\begin{tabular}{|l|c|c|c|}
\hline $\begin{array}{l}\text { Tabla \# } 3 \\
\text { Factores de comparación de los estándares y } \\
\text { protocolos para aplicaciones domóticas. }\end{array}$ & \multicolumn{2}{l|}{ Zigbee } \\
\hline \multicolumn{1}{|c|}{ Factores } & $\mathbf{X 1 0}$ & KNX & RF \\
\hline $\begin{array}{l}\text { Medios de } \\
\text { transmisión }\end{array}$ & Líneas de fuerza & $\begin{array}{c}\text { RF, Par trenzado, } \\
\text { líneas de fuerza }\end{array}$ & Media \\
\hline $\begin{array}{l}\text { Facilidad de } \\
\text { implementación }\end{array}$ & Media & Baja & Medio \\
\hline $\begin{array}{l}\text { Costos } \\
\text { Interoperabilidad }\end{array}$ & Bajo & Alto & $\begin{array}{c}\text { Entre marcas con el } \\
\text { estándar y otras } \\
\text { tecnologías }\end{array}$ \\
\hline $\begin{array}{l}\text { Aleance } \\
\text { Seguridad }\end{array}$ & $\mathrm{n} / \mathrm{a}$ & manejen el estándar & 60 m \\
\hline
\end{tabular}




\begin{tabular}{|c|c|c|c|}
\hline Fiabilidad & $\begin{array}{c}\text { Depende de línea } \\
\text { de corriente }\end{array}$ & Alta & Media \\
\hline $\begin{array}{l}\text { Cantidad de } \\
\text { dispositivos/ } \\
\text { Escalabilidad }\end{array}$ & $\mathrm{n} / \mathrm{a}$ & 64 por línea & $>100$ por red \\
\hline Servicios de control & $\begin{array}{c}\text { Aplicaciones } \\
\text { sencillas, control } \\
\text { limitado }\end{array}$ & $\begin{array}{c}\text { Todas las } \\
\text { aplicaciones del } \\
\text { hogar }\end{array}$ & $\begin{array}{c}\text { Todas las } \\
\text { aplicaciones del } \\
\text { hogar }\end{array}$ \\
\hline
\end{tabular}

Nota: RF: Radio Frecuencia, n/a: no aplica. Fuente, elaboración propia.

La interoperabilidad en todos los casos, a excepción de X10, está cubierta pues existe gran disponibilidad de marcas que implementan estos estándares, como el caso de ABB para KNX y Bticino para Zigbee (Bticino, 2014); por otro lado hay fabricantes que ofrecen tarjetas capaces de implementar Zigbee a un circuito desarrollado para algo puntual, lo que agrega cierto nivel de flexibilidad (Gaddam et al. 2011).

El rango de cobertura o alcance responde la necesidad de los hogares del Ecuador, donde la mayoría de proyectos habitacionales presentan viviendas con áreas de construcción entre $60 \mathrm{~m} 2$ y $120 \mathrm{~m} 2$ en la ciudad de Quito y de $50 \mathrm{~m} 2$ a $100 \mathrm{~m} 2$ en Guayaquil (Cámara de la Industria de la Construcción, 2014); por lo que el área que deben cubrir los estándares no es de gran tamaño y cualquiera de los aquí mencionados puede hacerlo sin inconvenientes.

En el ámbito de la seguridad al transmitir la información X10 es el único que no encripta los datos. Por otro lado la fiabilidad de transmisión de la información para lo que KNX funciona de la mejor manera cuando se utiliza cableado, X10 depende mucho de la calidad de las corrientes portadoras y Zigbee puede tener inconvenientes de atenuación debido a las paredes, vidrios o materiales que haya entre los dispositivos (Idoudi, Elkhorchani, y Grayaa, 2013) e incluso interferencia con otras tecnologías, como el caso de Wifi según la banda en la que trabaje (Langhammer, y Kays ,2012).

Con respecto a la simplicidad de implementación X10 y Zigbee ofrecen métodos sencillos, como dispositivos plug and play y autorregulación de la red, a pesar de ello se requiere tener cierto conocimiento de la estructura de la misma. Por otro lado KNX necesita la herramienta ETS para diseñar e implementar los sistemas, además a los requerimientos de cada fabricante para aprender a usar sus dispositivos; por lo que implica la intervención de un personal capacitado en el tema. Finalmente, con respecto a la capacidad para controlar las distintas utilidades en los hogares, Zigbee y KNX presentan una amplia gama de posibilidades mientras que X10 es utilizado para encendido y apagado de aplicaciones simples.

Los casos de éxito presentados anteriormente fueron identificados con letras para poder mostrar un resumen de sus características en la Tabla 4, la cual se divide en secciones relacionadas con la forma en la que se obtienen los datos, las funcionalidades propuestas para la seguridad de los adultos mayores, las interfaces para interacción con el usuario, la forma en que controlan el hogar, los estándares que manejan y la capacidad para trabajar con otras tecnologías. De esta forma es posible reconocer qué dispositivos utilizaban para cumplir el objetivo del sistema domótico del que forman parte. 


\section{Tabla \# 4}

\section{Proyectos y sus caracteristicas principales}

\begin{tabular}{|l|l|c|c|c|c|c|c|c|c|}
\hline & Características & A & B & C & D & E & F & G & H \\
\hline Estándar & Zigbee & Zigbee & Zigbee & $\begin{array}{c}\text { Zigbee/ } \\
\text { KNX }\end{array}$ & $\begin{array}{c}\text { Zigbee/ } \\
\text { Wifi }\end{array}$ & Zigbee & KNX & X10/ \\
Wifi
\end{tabular}

Nota: Fuente, elaboración propia

\section{Conclusiones}

En el presente trabajo se ha mostrado que el envejecimiento disminuye las capacidades físicas y mentales de las personas, limitando la independencia para realizar las actividades diarias y mermando su calidad de vida.

Al momento de escoger un estándar para implementar un sistema domótico se considerará que es una herramienta donde se hará posible dicho objetivo, mas no es el sistema en sí, pues este requiere una etapa de diseño basada en las necesidades de usuario, la arquitectura de la vivienda, los procesos a automatizar y las interfaces de usuario.

Luego de realizar la comparación bajo los parámetros propuestos, se muestra que $\mathrm{X} 10$ es un protocolo de muy bajo costo tanto en equipos como en instalación, no obstante es limitado al control de encendido y apagado, y depende de la calidad de las 
corrientes portadoras de la vivienda, lo que lo vuelve vulnerable.

KNX brinda gran fiabilidad, interoperabilidad con amplia gama de productos de marcas distintas, permite un sistema escalable, se puede utilizar para gestionar prácticamente todas las necesidades del hogar; pero tiene un elevado costo por mano de obra, costo de los dispositivos e implementación.

Tanto KNX como Zigbee son buenas opciones, pero en nuestro medio el factor costo le da una ventaja a Zigbee como el más idóneo para este tipo de proyectos, presenta ventajas de interoperabilidad y flexibilidad para crear circuitos ah-hoc para ciertas aplicaciones.

En base a los proyectos mostrados lo recomendable es una combinación de domótica de monitorización y control, pero como mínimo se propone utilizar una WSN, implementada con el estándar Zigbee, para recolectar datos y además otorgarle un control central para manejar sus electrodomésticos más usados.

La complejidad del sistema irá aumentando conforme a las necesidades de la persona y su capacidad para manejar la tecnología. En caso de ser posible, el uso de comandos de voz es recomendable, pues la interacción con el medio ocurre con mayor naturalidad y sencillez.

Teniendo en cuenta las limitaciones de la investigación al ser una revisión teórica, sus aplicaciones prácticas pueden ser validadas y enriquecidas, los proyectos presentados son de otros países pero aplicables a la realidad local. Se seleccionaron estándares representativos pero el que trabajen en medios de transmisión distintos influye en la comparación; no obstante se podrán hacer investigaciones posteriores que revisen otras opciones inalámbricas accesibles en nuestro medio.
Finalmente se indica que existen varias alternativas de estándares para sistemas domóticos además de los aquí presentados y es posible crear aplicaciones sencillas para simplificar la vida de los usuarios sin necesidad de altas inversiones, volviendo factible la oportunidad de contar con un ambiente inteligente, que mejore la vida, sobre todo la de los adultos mayores.

\section{Referencias Bibliográfica}

ABB (2014), ABB i-bus KNX, Intelligent installation systemas. System Description. Recuperado el 21 de julio del 2014 de http://www05.abb.com/global/scot/ scot 209 .nsf/veritydisplay/ e784bbf356160fcec125777e002aa94a/\$file/0156_systembe_gb_06_09.pdf

AlShu'eili, H., Sen Gupta, G., \& Mukhopadhyay, $\mathrm{S}$. (mayo, 2011). Voice recognition based wireless home automation system. In Mechatronics (ICOM), 2011 4th International Conference On (pp. 1-6). IEEE. doi:10.1109/1COM.2011.5937116

Arroyo, P., Lera, L., Sánchez, H., Bunout, D., Santos, J. L., \& Albala, C. (2007). Indicadores antropométricos, composición corporal y limitaciones funcionales en ancianos. Revista médica de Chile, 135(7), 846-854.

Barrantes-Monge, M., García-Mayo, E. J., Gutiérrez-Robledo, L. M., \& Miguel-Jaimes, A. (2007). Dependencia funcional y enfermedades crónicas en ancianos mexicanos. salud pública de México, 49, s459-s466. Recuperado en 21 de agosto de 2014, de http://www.scielo.org.mx/scielo.php?script =sci_arttex t\&pid=S0036-36342007001000004\&lng= es\&tlng $=$ es.

Bticino (2014). Catálogo línea MyHome. Recuperado el 21 de agosto del 2014 de http://www.bticino.cl/portal/web/catalogo-zigbee.php 
Collins, s. C., Bhatti, j. Z., Dexter, s. L. \& Rabbitt, p. M. (1992), Elderly people in a new world: attitudes to advanced communications technologies. In edited by $\mathrm{H}$. Boumqa and J. A. M. Graafmans Gerontechnology (Amsterdam: IOS Press).

Cámara de la Industria de la Construcción (2014). Evolución del Mercado de Vivienda en el Ecuador y Prospectiva de la Vivienda de Interés Social. Reuperado el 21 de julio de 2014 de http://www.camicon.ec/descargas_camicon/evolucion_del\%20_mercado_de\%20_ vivienda_en_el\%20ecuador.pdf

De Luca, G., Lillo, P., Mainetti, L., Mighali, V., Patrono, L., \& Sergi, I. (septiembre, 2013). The use of NFC and Android technologies to enable a KNX-based smart home. In Software, Telecommunications and Computer Networks (SoftCOM), 201321 st International Conference on (pp. 1-7). IEEE. doi:10.1109/SoftCOM.2013.6671904

Demiris, G., Rantz, M. J., Aud, M. A., Marek, K. D., Tyrer, H. W., Skubic, M., \& Hussam, A. A. (2004). Older adults ${ }^{\prime}$ attitudes towards and perceptions of smart home'technologies: a pilot study. Informatics for Health and Social Care, 29(2), 87-94. doi:10.1080/ 14639230410001684387

Domínguez, H. M., \& Vacas, F. S. (2006). Domótica: Un enfoque sociotécnico. Fundación Rogelio Segovia para el Desarrollo de las Telecomunicaciones.

Douligeris, C., Khawand, J., \& Khawand, C. (abril, 1991). Communications and control for a home automation system. In Southeastcon' 191., IEEE Proceedings of (pp. 171-175). IEEE. doi: 10.1109/ SECON.1991.147729
Dueñas Oviedo, N. (2012). Hogar multifuncional del adulto mayor : aprendizaje $y$ convivencia para la tercera edad activa. Facultad de Arquitectura, Diseño y Artes. PUCE. Quito. 2 v.

Edgar, J. A. (junio, 1987). Closed Loop Programmable Power a Smart House Innovation. In Telecommunications Energy Conference, 1987. INTELEC'87. The Ninth International (pp. 357-360). IEEE. doi: 10.1109/INTLEC.1987. 4794581

Fabbricatore, C., Zucker, M., Ziganki, S., \& Karduck, A. P. (mayo, 2011). Towards an unified architecture for smart home and ambient assisted living solutions: a focus on elderly people. In Digital Ecosystems and Technologies Conference (DEST), 2011 Proceedings of the 5th IEEE International Conference on (pp. 305-311). IEEE. doi: 10.1109/DEST.2011.5936643

Flores Marcillo, M. A., Yugsi, R., \& Elicio, R. (2014). Diseño e implementación de un sistema de seguridad con comunicación inalámbrica utilizando tecnología zigbee y control de eventos por medio de sms para la empresa de calzado Docceti Shoes.(tesis de pregrado, Universidad Politécnica Salesiana) Recuperado de http://dspace.ups.edu.ec/handle/ 123456789/6330

Gaddam, A, Mukhopadhyay, S.C. \& Gupta, G.S. (diciembre , 2009).Smart home for elderly care using optimized number of wireless sensors. Computers and Devices for Communication, 2009. CODEC 2009. 4th International Conference on, 1-4.

Gaddam, A., Mukhopadhyay, S. C., \& Sen Gupta, G. (mayo, 2011). Trial \& experimentation of a smart home monitoring system for elderly. In Instrumentation and Measurement Technology Conference (I2MTC), 2011 IEEE (pp. 1-6). IEEE. doi: 10.1109/IMTC.2011.5944230 
Gill, K., Yang, S. H., Yao, F., \& Lu, X. (2009). A zigbee-based home automation system. Consumer Electronics, IEEE Transactions on, 55(2), 422-430. doi: 10.1109/ TCE.2009.5174403

Hamabe, R., Murata, M., \& Namekawa, T. (1986). Home bus system (HBS) interface LSI and its standard protocol example. Consumer Electronics, IEEE Transactions on, (1), 9-19. doi: 10.1109/TCE. 1986.290112

Idoudi, M., Elkhorchani, H., \& Grayaa, K. (marzo,2013). Performance evaluation of Wireless Sensor Networks based on ZigBee technology in smart home. In Electrical Engineering and Software Applications (ICEESA), 2013 International Conference on (pp. 1-4). IEEE.

IEEE (septiembre, 2008).Standard Definitions and Concepts for Dynamic Spectrum Access: Terminology Relating to Emerging Wireless Networks, System Functionality, and Spectrum Management. IEEE Std 1900.1-2008, cl-48. doi: 10.1109/IEEESTD.2008.4633734

Junestrand S., Pasarte X., Vázquez D. (2005): Domótica y Hogar Digital, Thomson Paraninfo, Madrid.

KNX Association (2014). Tomado de http:// $\mathrm{knx} . o r g / \mathrm{knx}$-en/knx/association/introduction/index.php

KNX Association (2014). KNX TP1 Topology. Obtenido el 21 de julio de 2014 de http://www.knx.org/fileadmin/template/documents/downloads_support_ menu/KNX_tutor_seminar_page/basic_documentation/Topology E1212c.pdf

Koskela, T., \& Väänänen-Vainio-Mattila, K. (2004). Evolution towards smart home environments: empirical evaluation of three user interfaces. Personal and Ubiquitous Computing, 8(3-4), 234-240.
Langhammer, N., \& Kays, R. (2012). Performance evaluation of wireless home automation networks in indoor scenarios. Smart Grid, IEEE Transactions on,3(4), 2252-2261.

MIES(2012-2013). Agenda de igualdad para adultos mayores. Segunda Edición. Quito Ecuador

Mowafey, S., \& Gardner, S. (2013, october). Towards ambient intelligence in assisted living: The creation of an Intelligent Home Care. In Science and Information Conference (SAI), 2013 (pp. 51-60). IEEE.

MSP, (2011). Manual para cuidadores de la persona adulta mayor dependiente. Quito Ecuador

MSP y OPS, (2008). Guias clínicas geronto geriátricas de atención primaria de salud del adulto mayor. Quito Ecuador

Murata, M., Namekawa, T., \& Hamabe, R. (1983). A proposal for standardization of home bus system for home automation. Consumer Electronics, IEEE Transactions on, (4), 524-530. doi: 10.1109/TCE. 1983.356359

Nazabal, J.A., Matias, I.R., Fernández-Valdivielso, C., Falcone, F., Branchi, P. \& Mukhopadhyay, S.C. (diciembre, 2012).Home automation based sensor system for monitoring elderly people safety. Sensing Technology (ICST), 2012 Sixth International Conference, 142-145. doi: 10.1109/ICSensT.2012.6461658

Popescu, D., Dobrescu, R., Maciuca, A. \& Marcu, R. (noviembre, 2012).Smart sensor network for continuous monitoring at home of elderly population with chronic diseases. Telecommunications Forum (TELFOR), 603-606. doi:10.1109/ TELFOR.2012.6419282 
Raad, M.W. \& Yang, L.T. (julio, 2008). A ubiquitous smart home for elderly. Advances in Medical, Signal and Information Processing, 2008. MEDSIP 2008. 4th IET International Conference, 1-4.

Skrzypczak, C. (diciembre, 1987). The intelligent home of 2010. Communications Magazine, IEEE, 25(12),81-84. doi: 10.1109/MCOM.1987.1093504

Suryadevara, N.K., Mukhopadhyay, S. C., Rayudu, R.K. \& Huang, Y. M. (mayo, 2012).Sensor data fusion to determine wellness of an elderly in intelligent home monitoring environment. Instrumentation and Measurement Technology Conference (I2MTC), 2012 IEEE International, 947-952. doi: 10.1109/I2MTC. 2012.6229645

Suryadevara, N.K., Mukhopadhyay, S.C., Wang, R., Rayudu, R.K. \& Huang, Y.M. (Mayo, 2013).Reliable measurement of Wireless Sensor Network data for forecasting wellness of elderly at smart home. Instrumentation and Measurement Technology Conference (I2MTC), 2013 IEEE International, 16-21.

doi: 10.1109/I2MTC.2013.6555372

Synco (2013), KNX Bus communications. Device range Synco 700, Synco living, RXB/RXL, RDG, RDF, RDU. Basic Documentation.
Tritton, J. A. (junio, 1988). Interactive Home Systems (IHS)-an overview. Private Switching Systems and Net-works, 1988., International Conference, 195-200.

Viani, F., Robol, F., Polo, A, Rocca, P., Oliveri, G. \& Massa, A. (noviembre, 2013).Wireless Architectures for Heterogeneous Sensing in Smart Home Applications: Concepts and Real Implementation. Proceedings of the IEEE, 101(11), 2381-2396. doi: 10.1109/JPROC.2013.2266858

Withanage, C., Ashok, R., Chau Yuen \& Otto, K. (mayo, 2014).A comparison of the popular home automation technologies. Innovative Smart Grid Technologies Asia (ISGT Asia), 2014 IEEE, 600-605.doi: 10.1109/ISGT-Asia.2014. 6873860

Zigbee Alliance (2014). Recuperado de http://www.zigbee.org/Standards/Overview.aspx

\section{Ricardo Alarcón Contreras}

Ing. en Electrónica de la Universidad Espiritu Santo - UEES

E-mail: ralarcon@uees.edu.ec

\section{Mónica Flores Marin}

Ing. en Electricidad, Especialización Electrónica y Automatización Industrial. Magister en Administración de Empresas Docente tiempo completo de la Universidad Espiritu Santo - Ecuador

E-mail: mfloresm@gmail.com 